\title{
Heavy Metal Toxicity, Health Hazards and their Removal Technique by Natural Adsorbents: A Short Overview
}

\author{
Pournima R Pawar" ${ }^{*}$ and Shrikant M Bhosale ${ }^{\#}$ \\ \#Department of Technology, Shivaji University, Kolhapur, Maharashtra, India
}

Received 18 Feb 2018, Accepted 20 April 2018, Available online 26 April 2018, Vol.8, No.2 (March/April 2018)

\begin{abstract}
Environmental pollution particularly from heavy metals and minerals in the wastewater is the most severe problem in India. Due to extensive anthropogenic activities such as industrial operations particularly mining, agricultural processes and disposal of industrial waste materials; their concentration has increased to dangerous levels. Heavy metals in industrial effluent include Copper, nickel, chromium, lead, zinc, arsenic, cadmium, selenium and uranium. So far, a number of efficient methods have been reviewed for the removal of heavy metals such as chemical precipitation, ion exchange, reverse osmosis, electro dialysis, ultra filtration, nanofiltration, coagulation, flocculation, floatation, etc. However these methods have several disadvantages such as high reagent requirement, unpredictable metal ion removal, generation of toxic sludge etc. Adsorption process being very simple, economical, effective and versatile has become the most preferred methods for removal of toxic contaminants from wastewater. This paper reviews the use of various readily available natural materials as adsorbents of heavy metals from industrial wastewater. Various natural adsorbents reviewed includes sand, waste tea leaves eggshell, rice husk, activated carbon, zeolites, olive stones, wood sawdust etc.
\end{abstract}

Keywords: Heavy metals, adsorption, activated carbon, natural adsorbents.

\section{Introduction}

Water pollution caused due to addition of heavy metals resulting from the industrial activities is increasing tremendously and is a matter of global concern. Mining, mineral processing and metallurgical operations are generating effluents containing heavy metals. The heavy metals present in the wastewater is persistent and non degradable in nature. Moreover, they are soluble in aquatic environment and thus can be easily absorbed by living cells. Thus, by entering the food chain, they can be bio-accumulated and biomagnified in higher tropic levels also. The heavy metals, if absorbed above the permissible labels, could lead to serious health disorders. In light of the facts, treatment of heavy metals containing industrial effluent becomes quite necessary before being discharged into the environment. The scientists and environmental engineers are therefore facing a tough task of cost effective treatment of wastewater containing heavy metals. The conventional methods for heavy metal removal from wastewater includes chemical precipitation, chemical oxidation, ion exchange, membrane separation, reverse osmosis, electro dialysis etc. These methods are not very

*Correspoding author's ORCID ID: 0000-0002-0111-6166 DOI: https://doi.org/10.14741/ijcet/v.8.2.35 effective, are costly and require high energy input. They are associated with generation of toxic sludge, disposal of which renders it expensive and non ecofriendly in nature. In the recent past, number of approaches has been investigated for safe and economical treatment of heavy metal laden wastewater. Adsorption has emerged out to be better alternative treatment methods. It is said to be effective and economical because of its relatively low cost. The key benefit of adsorption method for heavy metal removal is less initial as well as operation cost unproblematic design and less requirement of control systems.

Generally the heavy metals are present in the wastewater at low concentrations and adsorption is suitable even when the metal ions are present at concentrations as low as $1 \mathrm{mg} / \mathrm{L}$. This makes adsorption an economical and favorable technology for heavy metal removal from wastewater. The adsorbent may be of mineral, organic or biological origin. It could be zeolites, industrial byproducts, agricultural waste, biomass and polymeric material. One of the conventional adsorbent, activated carbon has been extensively used in many applications. However, the high cost effectiveness of activation processes limits its usage in wastewater treatment processes. The present research activity aims toward contributing in the search for cost effective or natural adsorbents of 
natural origin and their applicability in recovery as well as removal of heavy metals from the industrial waste water.

\section{Toxicity of Heavy metals}

Metal toxicity or metal poisoning is the toxic effect of certain metals in certain forms and doses on life. Some metals are toxic when they form poisonous soluble compounds. Certain metals have no biological role, i.e. are not essential minerals, or are toxic when in a certain form. In the case of lead, any measurable amount may have negative health effects. Often heavy metals are thought as synonymous, but lighter metals may also be toxic in certain circumstances, such as beryllium and lithium. Not all heavy metals are particularly toxic, and some are essential, such as iron. The definition may also include trace elements when in abnormally high doses may be toxic. An option for treatment of metal poisoning may be chelation therapy, which is a technique which involves the administration of chelation agents to remove metals from the body.Toxic metals sometimes imitate the action of an essential element in the body, interfering with the metabolic process resulting in illness. Many metals, particularly heavy metals are toxic, but some heavy metals are essential, and some, such as bismuth, have a low toxicity. Most often the definition of toxic metals includes at least cadmium, manganese, lead, mercury and the radioactive metals. Radioactive metals have both radiological toxicity and chemical toxicity. Metals in an oxidation state abnormal to the body may also become toxic: chromium(III) is an essential trace element, but chromium(VI) is a carcinogen. Toxicity is a function of solubility. Insoluble compounds as well as the metallic forms often exhibit negligible toxicity. The toxicity of any metal depends on its ligands. In some cases, organometallic forms, such as methyl mercury and tetraethyl lead, can be extremely toxic. In other cases, organometallic derivatives are less toxic such as the cobaltocenium cation.

Decontamination for toxic metals is different from organic toxins: because toxic metals are elements, they cannot be destroyed. Toxic metals may be made insoluble or collected, possibly by the aid of chelating agents, or through bioremediation. Alternatively, they can be diluted into a sufficiently large reservoir, such as the sea, because immediate toxicity is a function of concentration rather than amount.

Toxic metals can bioaccumulate in the body and in the food chain. Therefore, a common characteristic of toxic metals is the chronic nature of their toxicity. This is particularly notable with radioactive heavy metals such as radium, which imitates calcium to the point of being incorporated into human bone, although similar health implications are found in lead or mercury poisoning. The exceptions to this are barium and aluminium, which can be removed efficiently by the kidneys.

\section{Human Health \& Hazards}

Heavy metal toxicity has proven to be a major threat and there are several health risks associated with it The toxic effects of these metals, even though they do not have any biological role, remain present in some or the other form harmful for the human body and its proper functioning. They sometimes act as a pseudo element of the body while at certain times they may even interfere with metabolic processes. Few metals, such as aluminium, can be removed through elimination activities, while some metals get accumulated in the body and food chain, exhibiting a chronic nature. Various public health measures have been undertaken to control, prevent and treat metal toxicity occurring at various levels, such as occupational exposure, accidents and environmental factors. Metal toxicity depends upon the absorbed dose, the route of exposure and duration of exposure, i.e. acute or chronic. This can lead to various disorders and can also result in excessive damage due to oxidative stress induced by free radical formation. This review gives details about some heavy metals and their toxicity mechanisms, along with their health effects.

In the industrialization era, pollution has deteriorated the quality and diversity of aquatic life. Industries discharge their waste containing heavy metals into the water bodies. These heavy metals accumulate in different organs of the fish, causing mortality. This effect first appears in blood and affects the fish by alteration of blood components making the fish weak, anemic and vulnerable to diseases. Heavy metals pollution is thus targeting the major protein source in the form of fish. The exposures to heavy metals cause increase or decrease in hematological indices, with decline in protein and glycogen reserves. Therefore the impact of heavy metals was carried out on the fish Labeorohita. Heavy metals affect the reticulo endothelial system and haematopoisis, changing osmotic resistance of erythrocytes. The red and white blood cells at different stages of the pathological process are subjected to quantitative and qualitative deformation.

\section{Source of waste heavy metals}

The two main sources of heavy metals in water and wastewater are natural and human. The natural factors include soil erosion, volcanic activities, urban run offs and aerosols particulate while the human factors include metal finishing and electroplating processes, mining extraction operations, textile industries and nuclear power. Then main natural sources of heavy metal pollutants in wastewater effluents are volcanic activities, soil erosion, urban run offs and aerosol particles. It is reported that volcanic eruptions produce hazardous impacts to the environment, climate and health of exposed individuals. Apart from the deterioration of social and chemical conditions and the gases (carbon dioxide, sulphur dioxide, carbon 
monoxide, hydrogen sulphide) released during eruptions, various organic compounds and heavy metals, such as mercury, lead and gold are also released. The presence of these heavy metals in water bodies is known to significantly deteriorate the quality of such waters. Several rocks and volatiles of volcanic origins are indicated to be responsible for the presence of metals in soils and waters. This is because the diffusion of acidic volcanic gases through water permeable rocks contributes to the hydrological material transfer in volcanic strata. The activities from volcanoes are reported to be responsible for the release of metals such as arsenic, mercury, aluminum, rubidium, lead, magnesium, copper, zinc and a host of others. Soil erosion is also indicated to be a source of heavy metal pollution in water. The two main agents of soil erosion are wind and water. During rainfall, sediment-bound heavy metals is distributed to the soil. Water containing agrochemicals with toxic metal concentration drop this sediment bound metal in the soil even as it causes erosion. During run-off due to erosion, heavy metals, may be picked up and distributed to the environment. In some cases, during rainfall, some heavy metal wastes are washed into poor drainage systems and subsequently into nearby rivers. In addition, some aerosol (fine colloidal particles or water droplet in the air, in some cases they can be gas) particles may carry different kinds of contaminant; like smoke cloud and heavy metals. These heavy metal containing aerosols usually accumulate on leaf surfaces in the form of fine particulates and can enter the leaves via stomata. Some of the human sources of heavy metals in wastewater effluents are metal finishing and electroplating, mining and extraction operations, textiles activities and nuclear power. Metal finishing and electroplating involve the deposition of thin protective layers into prepared surfaces of metal using electrochemical processes.

When this happens, toxic metals may be released into wastewater effluents. This may be either through rinsing of the product or spillage and dumping of process baths. It is also indicated that the cleaning of process tanks and treatment of wastewater can generate substantial quantities of wet sludge containing high levels of toxic metals. Similarly, mining activities can release toxic metals to the environment. Metal mining and smelting activities are regarded as major sources of heavy metals in the environment. In environments where these activities take place, it is indicated that large amount of toxic metals deposits are found in their water, soil, crops and vegetable.

Additionally, textile industries are indicated to be major sources of heavy metal pollutants in water. This is said to mostly originate from the dyeing process, which is a major process in such industries. The compounds used for these dyeing processes (coloration) include copper, chromium, nickel and lead which is very toxic and carcinogenic. In some cases, nuclear generating facilities have also been described as source of discharge of heavy metals like copper and zinc to surface water. In the nuclear plants large amount of water is consumed.

\section{Need for the removal of heavy metals}

Continuous discharge of industrial, domestic and agricultural wastes in rivers and Lakes causes deposit of pollutants in sediments. Such pollutants include heavy metals, which endanger public health after being incorporated in food chain. Heavy metals cannot be destroyed through biological degradation, as is the case with most organic Pollutants. Incidence of heavy metal accumulation in fish, oysters, mussels, sediments and other components of aquatic ecosystems have been reported from all over the World. Excessive amounts of some heavy metals can be toxic through direct action of the metal or through their inorganic salts or via organic compounds from which the metal can become easily detached or introduced into the cell. Exposure to different metals may occur in common circumstances, particularly in industrial setting. Accidents in some environments can result in acute, high level exposure. Some of the heavy metals are toxic to aquatic organisms even at low concentration. The problem of heavy metal Pollution in water and aquatic organisms including fish, needs continuous monitoring and surveillance as these elements do not degrade and tend to biomagnified in man through food chain. Hence there is a need to remove the heavy metals from then Aquatic ecosystems .Research and development, therefore focuses on sector-specific methods and Technologies to remove colour and heavy metals from different kinds of waste Streams. In view of the above toxicological effects of heavy metals on environment, Animals and human beings, it becomes imperative to treat these toxic compounds in Wastewater effluents before they are discharged into freshwater bodies.

\section{Heavy metal removal methods}

\section{A) Physico-chemical methods}

Following methods have been used by various researchers for removal of heavy metals. Physical separation techniques are primarily applicable to particulate forms of metals, discrete particles or metal bearing particles. Physical separation consists of mechanical screening, hydrodynamic classification, gravity concentration, flotation, magnetic separation, electrostatic separation, and attrition scrubbing, The efficiency of physical separation depends on various soil characteristics such as particle size distribution, particulate shape, clay content, moisture content, humic content, heterogeneity of soil matrix, density between soil matrix and metal contaminants, magnetic properties, and hydrophobic properties of particle surface.

\section{B) Chemical Precipitation}

Chemical precipitation is one of the most widely used for heavy metal removal from inorganic effluent in 
industry due to its simple operation. These conventional chemical precipitation processes produce insoluble precipitates of heavy metals as hydroxide, sulfide, carbonate and phosphate. The mechanism of this process is based on to produce insoluble metal precipitation by reacting dissolved metals in the solution and precipitant. In the precipitation process very fine particles are generated and chemical precipitants, coagulants, and flocculation processes are used to increase their particle size to remove them as sludge. Once the metals precipitate and form solids, they can easily be removed, and low metal concentrations, can be discharged. Removal percentage of metal ions in the solution may be improved to optimum by changing major parameters such as $\mathrm{pH}$, temperature initial concentration, charge of the ions etc. The most commonly used precipitation technique is hydroxide treatment due to its relative simplicity, natural of precipitant (lime), and ease of automatic $\mathrm{pH}$ control. The solubilities of the various metal hydroxides are minimized for $\mathrm{pH}$ in the range of 8.0 to 11.0.

\section{C) Coagulation and Flocculation}

The coagulation-flocculation mechanism is based on zeta potential $(\zeta)$ measurement as the criteria to define the electrostatic interaction between pollutants and coagulant-flocculant agents. Coagulation process is reduced the net surface charge of the colloidal particles to stabilize by electrostatic repulsion process. Flocculation process continually increases the particle size to discrete particles through additional collisions and interaction with inorganic polymers formed by the organic polymers added. Once discrete particles are flocculated into larger particles, they can be removed or separated by filtration, straining or floatation. Production of sludge, application of chemicals and transfer of toxic compounds into solid phase are main drawbacks of this process.

\section{D) Electrochemical Treatments}

Electrolytic recovery is one technology used to remove metals from wastewater streams. This process uses electricity to pass a current through an aqueous metalbearing solution containing a cathode plate and an insoluble anode. Electricity can be generated by movements of electrons from one element to another. Electrochemical process to treat wastewater containing heavy metals is to precipitate the heavy metals in a weak acidic or neutralized catholyte as hydroxides. Electrochemical treatments of wastewater involve electro-deposition, electro-coagulation, electroflotation and electro-oxidation. Electrode stabilization of colloids is called coagulation and precipitation by hydroxide formation to acceptable levels. It is the most common heavy metal precipitation method forming coagulants by electrolytic oxidation and destabilizing contaminants to form folc. The electro-coagulation process the coagulant is generated in situ by electrolytic oxidation of an appropriate anode material. In this process, charged ionic metal species are removed from wastewater by allowing it to react with anion in the effluent. This process is characterized by reduced sludge production, no requirement for chemical use, and ease of operation. However, chemical precipitation requires a large amount of chemicals to reduce metals to an acceptable level for discharge. Other drawbacks are huge sludge production, slow metal precipitation, poor settling, the aggregation of metal precipitates, and the long-term environmental impacts of sludge disposal. It changes the aqueous pollution problem to a solid waste disposal problem without recovering the metal.

\section{E) Ion Exchange}

Ion exchange can attract soluble ions from the liquid phase to the solid phase, which is the most widely used method in water treatment industry. As a cost-effective method, ion exchange process normally involves lowcost materials and convenient operations, and it has been proved to be very effective for removing heavy metals from aqueous solutions, particular for treating water with low concentration of heavy metals. In this process cations or anions containing special ion exchanger is used to remove metal ions in the solution. Commonly used ion exchangers are synthetic organic ion exchange resins. It can be used only low concentrated metal solution and this method is highly sensitive with the $\mathrm{pH}$ of the aqueous phase. Ion exchange resins are water-insoluble solid substances which can absorb positively or negatively charged ions from an electrolyte solution and release other ions with the same charges into the solution in an equivalent amount. The positively charged ions in cationic resins such as hydrogen and sodium ions are exchanged with positively charged ions, such as nickel, copper and zinc ions, in the solutions. Similarly, the negative ions in the resins such as hydroxyl and chloride ions can be replaced by the negatively charged ions such as chromate, sulfate, nitrate, cyanide and dissolved organic carbon (DOC).

\section{F) Membrane Filtration}

Membrane filtration has received considerable attention for the treatment of inorganic effluent. It is capable of removing suspended solid, organic compounds and inorganic contaminants such as heavy metals. Depending on the size of the particle that can be retained, various types of membrane filtration such as ultra filtration, nanofiltration and reverse osmosis can be employed for heavy metal removal from wastewater.

1) Ultra filtration (UF) utilizes permeable membrane to separate heavy metals, macromolecules and suspended solids from inorganic solution on the basis of the pore size (5-20 nm) and molecular weight of the 
separating compounds (1000-100,000 Da). Depending on the membrane characteristics, UF can achieve more than $90 \%$ of removal efficiency with a metal concentration ranging from 10 to $112 \mathrm{mg} / \mathrm{L}$ at $\mathrm{pH}$ ranging from 5 to 9.5 and at 2-5 bar of pressure. UF presents some advantages such as lower driving force and a smaller space requirement due to its high packing density.

2) Polymer-supported ultra filtration (PSU) technique adds water soluble polymeric ligands to bind metal ions and form macromolecular complexes by producing a free targeted metal ions effluent .Advantages of the PSU technology are the low-energy requirements involved in ultra filtration, the very fast reaction kinetics and higher selectivity of separation of selective bonding agents in aqueous solution. Another similar technique, complexation- ultra filtration, proves to be a promising alternative to technologies based on precipitation and ion exchange. The use of water-soluble metal-binding polymers in combination with ultra filtration (UF) is a hybrid approach to concentrate selectively and to recover heavy metals in the solution. In the complexation - UF process cationic forms of heavy metals are first complexed by a macroligand in order to increase their molecular weight with a size larger than the pores of the selected membrane. The advantages of complexation-filtration process are the high separation selectivity due to the use of a selective binding and low-energy requirements involved in these processes. Water-soluble polymeric ligands have shown to be powerful substances to remove trace metals from aqueous solutions and industrial wastewater through membrane processes.

3) Reverse osmosis (RO) is a separation process that uses pressure to force a solution through a membrane that retains the solute on one side and allows the pure solvent to pass to the other side. The membrane here is semi-permeable, meaning it allows the passage of solvent but not for metals. The membranes used for reverse osmosis have a dense barrier layer in the polymer matrix where most separation occurs. Reverse osmosis can remove many types of molecules and ions from solutions, including bacteria, and is used in both industrial processes. Reverse osmosis involves a diffusive mechanism, so that separation efficiency is dependent on solute concentration, pressure, and water flux rate.

\section{G) Electrodialysis}

Electrodialysis (ED) is a membrane separation in which ionized species in the solution are passed through an ion exchange membrane by applying an electric potential. The membranes are thin sheets of plastic materials with either anionic or cationic characteristics. When a solution containing ionic species passes through the cell compartments, the anions migrate toward the anode and the cations toward the cathode, crossing the anion exchange and cation-exchange membranes. A noticeable disadvantage is membranes replacement and the corrosion process. Using membranes with higher ion exchange capacity resulted in better cell performance. Effects of flow rate, temperature and voltage at different concentrations using two types of commercial membranes, using a laboratory ED cell, on lead removal were studied. Results show that increasing voltage and temperature improved cell performance and separation percentage decreased with an increasing flow rate. This offers advantages for the treatment of highly concentrated wastewater laden with heavy metals to recovery undesirable impurities from water.

\section{H) Biological Methods (Natural)}

Biological removal of heavy metals in water and wastewater involves the use of biological techniques for the elimination of pollutants from water and wastewater. In this processes micro-organisms play a role of settling solids in the solution.

1) Activated sludge, trickling filters, stabilization ponds are widely used for treating wastewater. Activated sludge is the most common option uses microorganisms in the treatment process to break down organic material with aeration and agitation, and then allows solids to settle out. Bacteria containing "activated sludge" is continually re-circulated back to the aeration basin to increase the rate of organic decomposition. Most of the research on heavy metals removal in biological systems has been directed towards the suspended growth activated sludge process. Trickling Filters which consist beds of coarse media (often stones or plastic) 3-10 ft. deep help to grow microorganisms. Wastewater is sprayed into the air (aeration), then allowed to trickle through the media and microorganisms break down organic materials in the wastewater. Trickling filters drain at the bottom and the wastewater is collected and then undergoes sedimentation. Stabilization ponds or lagoons are slow, cheap, and relatively inefficient, biological method that can be used for various types of wastewater. They rely on the interaction of sunlight, algae, microorganisms, and oxygen.

2) Biosorption is another method that can use to remove heavy metals from wastewater. Sorption process is transfer of ions from solution phase to the solid phase, actually describes a group of processes, which includes adsorption and precipitation reactions. Adsorption has become one of the alternative treatment techniques for water and wastewater. Basically, adsorption is a mass transfer process and substances bound by physical and or chemical interactions to solid surface. Various natural adsorbents, derived from agricultural waste, industrial by-product, natural material, or modified biopolymers, have been recently developed and applied for the removal of heavy metals from metal-contaminated water. Use of activated carbon in water and wastewater treatment has been oriented towards organics removal. 
Research efforts on inorganics removal by activated carbon, specifically metallic ions, have been clearly limited .Selective adsorption by red mud, coal, photocatalyst beads , nano-particles fertilizer industrial waste, biomass, activated sludge biomass , algae etc. has generated increasing excitement. Industrial by-products such as fly ash, waste iron, iron slags, hydrous titanium oxide, can be chemically modified to enhance its removal performance for metal removal from wastewater. Recently, research for the removal of heavy metals from industrial effluent has been focused on the use of agricultural by-products as adsorbents through biosorption process. New resources such as hazelnut shell, rice husk, pecan shells, jackfruit, maize cob or husk, rice straw, rice husk, coconut shell etc can be used as an adsorbent for heavy metal uptake after chemical modification or conversion by heating into activated carbon or biochar. They found that the maximum metal removal occurred by those biomass due to containing of cellulose, lignin, carbohydrate and silica in their adsorbent.

Biopolymers are posse a number of different functional groups, such as hydroxyls and amines, which increase the efficiency of metal ion uptake. They are widely use in industrially as they are capable of lowering transition metal ion concentrations to subpart per billion concentrations. New polysaccharide based-materials are described as biopolymer adsorbents (derived from chitin, chitosan, and starch) for the removal of heavy metals from the wastewater. The sorption mechanisms of polysaccharide-based materials are complicated and depend on $\mathrm{pH}$. Also hydrogels, which are cross linked hydrophilic polymers, are widely used in the purification of wastewater. The removal is basically governed by the water diffusion into the hydrogel , carrying the heavy metals inside especially in the absence of strongly binding sites. Maximum binding capacity increases with higher $\mathrm{pH}$ due to polymerization/cross linking reaction.

\section{Various factors affecting adsorption capacity}

The most important factors affecting adsorption are:

- Surface area of adsorbent. Larger sizes imply a greater adsorption capacity.

- Particle size of adsorbent. Smaller particle sizes reduce internal diffusional and mass transfer limitation to the penetration of the adsorbate inside the adsorbent (i.e., equilibrium is more easily achieved and nearly full adsorption capability can be attained). However, wastewater drop across columns packed with powdered material is too high for use of this material in packed beds. Addition of powdered adsorbent must be followed by their removal.

- Contact time or residence time. The longer the time the more complete the adsorption will be. However, the equipment will be larger.

- Solubility of solute (adsorbate) in liquid (wastewater). Substances slightly soluble in water will be more easily removed from water (i.e., adsorbed) than substances with high solubility. Also, non-polar substances will be more easily removed than polar substances since the latter have a greater affinity for water.

- Affinity of the solute for the adsorbent (carbon). The surface of activated carbon is only slightly polar. Hence non-polar substances will be more easily picked up by the carbon than polar ones.

- Number of carbon atoms. For substances in the same homologous series a larger number of carbon atoms is generally associated with a lower polarity and hence a greater potential for being adsorbed (e.g., the degree of adsorption increases in the sequence formicaceticpropionic-butyric acid).

\section{Evaluation of heavy metals removal processes}

In general, physico-chemical treatments offer various advantages such as their rapid process, ease of operation and control, flexibility to change of temperature. There are numerous methods currently employed to remove and recover the metals from our environment and many physico-chemical methods have been proposed for their removal from wastewater. These include chemical oxidation and reduction, membrane separation, liquid extraction, carbon adsorption, ion exchange, electrolytic treatment, electro precipitation, coagulation, flotation, evaporation, hydroxide and sulfide precipitation, crystallization, ultra filtration, electro dialysis etc. . These methods differ in their effectiveness and cost Chemical precipitation, reverse osmosis and other methods (ultra filtration, electrochemical deposition etc.) become inefficient when contaminants are present in trace concentration. And do not seem to be economically feasible for such industries because of their relative high costs. Therefore, there is a need to look into alternatives to investigate a low-cost method which is effective and economic. For high strength and low volumes of wastewater, heavy metal removal by adsorption technique is good proposition. Adsorption is one of the alternatives for such cases and is an effective purification and separation technique used in industry especially in water and wastewater treatments. It is the tendency of molecules from an ambient fluid phase to adhere to the surface of a solid. Adsorption has advantages over other methods. The design is simple, and it is sludge-free and can involve low investment in terms of both the initial costs and land. Cost is an important parameter for comparing the adsorbent materials. Activated carbon has been recognized as a highly effective adsorbent for the treatment of heavy metals in wastewater, but is readily soluble under extreme $\mathrm{pH}$ conditions. Activated carbon is most widely used adsorbent, as it has good capacity for adsorption of carcinogenic metals. However, high cost of activated carbon and 10-15\% loss during the regeneration has deterrents in the utilization of activated carbon but is replaced natural adsorbent for the developing countries. 


\section{Conclusions}

A review of various processes and adsorbents for heavy metal removal shows that adsorption process has great potential for the elimination of heavy metals from water and wastewater using natural adsorbents. More studies should be carried out for low-cost adsorption process to promote large scale use of nonconventional adsorbents. Natural adsorbents should be used to minimize cost and maximize heavy metal removal efficiency

The role of natural product in the removal of heavy metals ions from water and wastewater has been reviewed. The adsorption of heavy metal ions on the adsorbent studied by various researchers could be influenced by a number of operating conditions, such as, initial metal ion concentration, solution $\mathrm{pH}$, contact time, adsorbent dose, temperature and on the ionic strength of the aqueous solution. Generally, removal of metal ions increased with increased adsorbent dose and contact time. However, favourable adsorption conditions may be different for different adsorbents and metal ions.

If it is possible to develop such biosorbents from natural product, then these adsorbents may offer significant advantages over currently available commercially expensive activated carbons, and in addition contribute to an overall waste minimization strategy.
Further more studies are required to apply the design and simulation model to larger scale pilot plant and not on small scale laboratory applications.

\section{References}

Dimple Lakherwal. (2014), Adsorption of Heavy Metals: A Review International Journal of Environmental Research and Development. Volume 4, Number 1 (2014), pp. 4148.

AshutoshTripathi* and ManjuRawatRanjan (2015), Heavy Metal Removal from Wastewater Using Low Cost Adsorbents, Journal of Bio remediation \& Bio degradation DOI: $10.4172 / 2155-6199.1000315$.

Alkesh I. Shah . (2017), Heavy metal impact on aquatic life and human health - an over view, International Journal of Environmental Research and Devel37th Annual Conference of the International Association for Impact Assessment.

Jamal A Radaideh1,Hassan Al Abdulgader ,Matthias Barjenbruch. (2017), Evaluation of Absorption Process for Heavy Metals Removal found in Pharmaceutical Wastewater, Journal of Medical Toxicology and Clinical Forensic Medicine.Vol.3 No.2:9

Gunatilake S.K. (2015), Methods of Removing Heavy Metals from Industrial Wastewater, Journal of Multidisciplinary Engineering Science Studies .Vol. 1 Issue 1, November 2015.

Oghenerobor Benjamin Akpor, Gladys OnolunoseOhiobor, and Tomilola Debby Olaolu. (2014), Heavy metal pollutants in wastewater effluents: Sources,effects and remediation, Advances in Bioscience and Bioengineering. Vol. 2, No. 4 , 2014, pp. 37-43. 